\title{
Antineoplastic drug contamination in the urine of Canadian healthcare workers
}

\author{
Chun-Yip Hon, Kay Teschke, Hui Shen, Paul A. Demers, and \\ Scott Venners
}

Version Post-Print/Accepted Manuscript

Citation Hon C-Y, Teschke K, Shen H, Demers PA, Venners S. Antineoplastic

(published version) Drug Contamination in the Urine of Canadian Workers. Int Arch

Occup Environ Health 2015 Oct;88(7):933-41. PMID 25626912.

Publisher's Statement The final publication is available at Springer via

http://dx.doi.org/10.1007/s00420-015-1026-1.

Always cite the published version, so the author(s) will receive recognition through services that track citation counts, e.g. Scopus. If you need to cite the page number of the TSpace version (original manuscript or accepted manuscript) because you cannot access the published version, then cite the TSpace version in addition to the published version using the permanent URI (handle) found on the record page. 
Antineoplastic drug concentration levels in the urine of Canadian healthcare workers

Chun-Yip Hon ${ }^{1,2}$

Kay Teschke ${ }^{1}$

Hui Shen ${ }^{1}$

Paul A Demers ${ }^{3,4}$

Scott Venners ${ }^{5}$

${ }^{1}$ School of Population and Public Health, University of British Columbia

${ }^{2}$ School of Occupational and Public Health, Ryerson University

${ }^{3}$ Occupational Cancer Research Centre, Cancer Care Ontario

${ }^{4}$ Dalla Lana School of Public Health, University of Toronto

${ }^{5}$ Faculty of Health Sciences, Simon Fraser University

Corresponding Author:

Chun-Yip Hon

School of Occupational and Public Health

Ryerson University

350 Victoria Street, POD $247 C$

Toronto, Ontario

M5B $2 K 3$

Email:cyhon@ryerson.ca 


\begin{abstract}
Purpose: The purpose of this study was to quantify the urine concentration of non-metabolized cyclophosphamide (CP), a commonly administered antineoplastic drug, among potentially exposed Canadian healthcare workers and to identify factors associated with the drug concentration levels. Methods: Participants were asked to provide two sets of 24-hour urine samples (at two different sampling events) and the level of CP was quantified using highperformance liquid chromatography-tandem mass spectrometry. In addition to demographic information, participants were surveyed regarding their frequency of handling of antineoplastic drugs, safe drug handling training, and known contact with CP on their work shift. Descriptive and inferential statistical analyses were performed. A backward stepwise linear mixed effect model was conducted to identify factors associated with urine concentration levels. Results: We collected 201 urine samples and 55\% $(\mathrm{n}=111)$ had levels greater than the LOD of $0.05 \mathrm{ng} / \mathrm{mL}$. The mean urinary $\mathrm{CP}$ concentration was $0.156 \mathrm{ng} / \mathrm{mL}$, the geometric mean was $0.067 \mathrm{ng} / \mathrm{mL}$, the geometric standard deviation was 3.18 , the $75^{\text {th }}$ percentile was $0.129 \mathrm{ng} / \mathrm{mL}$ and the range was <LOD to $2.37 \mathrm{ng} / \mathrm{mL}$. All eight job categories evaluated had some urinary concentrations in excess of the LOD with unit clerks having the highest average level. Workers who worked in the drug administration unit but were not responsible for administering the drugs to patients, i.e. volunteers, oncologists, ward aides and dieticians, had the largest proportion of samples exceeding the LOD. We did not find any correlation between the urinary concentration levels and known contact with $\mathrm{CP}$ during the work shift. Two factors were found to be significantly associated with urinary $\mathrm{CP}$ concentration: (1) workers who had a duty to handle antineoplastic drugs had higher concentration levels; (2) workers who had not received safe drug handling training had higher levels of CP in their urine compared to those who had. Conclusions: The
\end{abstract}


presence of non-metabolized $\mathrm{CP}$ in urine confirms that, despite the existence of control measures, a broad range of healthcare workers are at risk of exposure to antineoplastic drugs. A review of the effectiveness of interventions to reduce exposure is warranted and should apply to all healthcare workers involved in some capacity with the hospital medication system. This study identified two factors that are related to the urine $\mathrm{CP}$ concentration levels which can serve as an impetus for reducing exposure.

\section{Key words}

Antineoplastic drugs, urinary concentration, healthcare workers, hospital medication system, risk assessment 


\section{Introduction}

Health risks associated with healthcare workers' exposure to antineoplastic drugs have been known since the 1970's (NIOSH 2004). Associated adverse health outcomes include mutagenicity, reproductive toxic effects and cancer (Dranitsaris et al. 2005; Ritchie et al., 2000; Connor and McDiarmid 2006). The primary route of occupational exposure to antineoplastic drugs has been shown to be to be through dermal contact (Sessink et al. 1994; Fransman et al. 2005). This can occur either: a) directly through handling of the drug vials or intravenous preparations or b) indirectly by contacting drug-contaminated surfaces/objects. We previously reported that healthcare workers are at risk of exposure to antineoplastic drugs by confirming the presence of drug contamination on the hands of pharmacy personnel (Hon et al. 2011a). In a more recent study, we demonstrated that an assortment of healthcare job categories throughout the hospital medication system (process flow of drugs within a facility from initial delivery to eventual disposal) have measurable dermal concentration levels (Hon et al. 2014b)

The collection of dermal wipes is practical because they are non-invasive; however, they are only representative of the level of external contamination and do not account for drug that may have been absorbed by an individual. The absorbed dose is important because most antineoplastic drugs, including the marker drug in our study, cyclophosphamide (CP), are initially inactive and do not exhibit cytostatic effects until they are metabolized (de Jonge et al. 2005). Furthermore, dermal wipe samples do not account for other potential routes of occupational exposure such as inhalation or ingestion and, therefore, may not provide a complete measure of exposure. Given this, a number of occupational exposure studies have collected urine samples as a biomarker to estimate the dose of antineoplastic drug as evidenced in reviews conducted by Suspiro and Prista (2011) and Hon et al. (2014a). 
The aim of this study was to assess the concentration of non-metabolized $\mathrm{CP}$ in the urine of a broad range of Canadian healthcare workers who are potentially exposed to antineoplastic drugs due to their role in the hospital medication system. In addition, we sought to identify individual and workplace factors that may be associated with the concentration of $\mathrm{CP}$ in urine.

\section{Methodology}

\section{Selection of participants}

Ethics approval was obtained prior to study commencement. Six facilities participated in the study - five acute care sites and one cancer treatment centre. Additional characteristics of the participating facilities have been described previously (Hon et al. 2011b) Participants were selected from those who were considered potentially exposed following site observations to identify drug-contaminated surfaces and the job categories that contacted the suspect surfaces. The job categories selected for inclusion were pharmacists, pharmacy receiver, pharmacy technician, nurse, transport staff, unit clerks and others working in drug administration units (volunteers, ward aides, oncologists and dieticians). The participating hospitals' research ethics boards dictated the form of recruitment. Participants were selected either actively through a mailed letter of invitation or passively via the distribution of consent to contact forms at departmental meetings. For each job category, up to three representatives per site were invited to participate.

Upon receiving consent from workers to participate in the study, members of the research team contacted each participant via email or telephone to arrange a mutually convenient time to meet at their place of work to collect samples. Every participant was given a cash honorarium of $\$ 25$ for providing a urine sample. 


\section{Collection of urine samples}

As elimination of CP is almost complete 24 hours after uptake (de Jonge et al. 2005), participants were asked to provide 24-hour urine samples. This same sampling period is recommended by the U.S. Environmental Protection Agency for biological monitoring of toxic substances (US EPA 2011) and is also consistent with other urinary biomarker studies of anticancer agents (Suspiro and Prista 2011; Jakubowski and Trzcinka-Ochocka 2005).

Participants were asked to provide the initial urine sample immediately after the dermal wipe was collected (convenient sampling during the course of a work shift) (Hon et al. 2014b) Every participant was supplied with ten $250 \mathrm{~mL}$ polypropylene collection jars (Fisher Scientific, Ottawa, ON) with every void during the 24 -hour period collected in a new jar. To prevent potential degradation, participants were asked to keep the urine samples refrigerated whenever practical and, where a refrigerator was unavailable, participants were asked to use the instant cold packs provided to facilitate the maintenance of a colder environment.

Once the 24-hour sampling period was completed, the samples were couriered to the Occupational and Environmental Hygiene laboratory at the University of British Columbia. All urine samples from a participant (typically between 6 and 7 voids in the 24-hour period) were pooled and three 5-mL aliquots of the 24-hr pooled sample were placed into cryogenic tubes and subsequently stored at $-80^{\circ} \mathrm{C}$ until analysis.

A second set of 24-hour urine samples was collected from most participants with at least three weeks' lag between collection times (average of 97 days; range 22 to 188 days). Participants were not made aware of the results from the first collection date before the second sample was collected. Sample collection took place between June 2010 and February 2011.

Urine sample analysis 
For every participant's sample, one of the $5 \mathrm{~mL}$ aliquots was allowed to thaw. After thawing, contents were transferred to a scintillation vial and an internal standard, $50 \mu \mathrm{L}$ of D4-CP (0.05 ng/ $\mu$ l) (Toronto Research Chemicals, Toronto, Canada), was added. Ethyl acetate solvent (Sigma Aldrich, Oakville, ON) was added to separate the organic matter from the aqueous layer. This step was repeated three times until virtually all the organic matter was extracted. The culture tube containing organic matter was then allowed to dry under a gentle stream of nitrogen gas. Once dry, the residual was reconstituted in $1.0 \mathrm{~mL} 0.1 \mathrm{M}$ ammonium acetate (Sigma Aldrich, Oakville, ON) and this amount was transferred to liquid chromatography vials.

The aliquot was analyzed for CP by high-performance liquid chromatography-tandem mass spectrometry using an Agilent Technologies 6410 Triple Quadruple LC-MS/MS (Santa Clara, CA). The instrument employed a Zorbax XDB-C18 column (Agilent Technologies, Santa Clara, CA) with a gradient mobile phase of $5 \mathrm{mM}$ Ammonium Acetate:100\% Methanol (A:B) (Sigma Aldrich, Oakville, ON) and samples were run at a flow rate of $0.5 \mathrm{~mL} / \mathrm{min}$. A 10-point calibration curve was used and, for quality control purposes, a calibration standard was run for every 10 samples. For additional quality control, QC spike samples, urine spike samples and blanks were included in the analysis. Ten percent of the samples in each batch were run in duplicate and a duplicate response that varied by more than $10 \%$ was not tolerated ${ }^{1}$. The limit of detection (LOD) was 0.05 nanograms per milliliter $(\mathrm{ng} / \mathrm{mL})$ and the urinary drug concentrations were reported in $\mathrm{ng} / \mathrm{mL}$.

\section{Supplemental data collected}

We collected a variety of information from the participants while on-site as well as via a selfadministered questionnaire. After the urine sample was collected, all participants were surveyed

\footnotetext{
${ }^{1}$ If duplicate response varied by more than $10 \%$, the batch was re-analyzed to see if the instrument was an issue or the extraction. Some samples were re-run and the resulting concentrations were used for this study.
} 
by a member of the research team regarding the type and frequency of contact with $\mathrm{CP}$ during the current work shift. Types of contact with CP included mixing, administering, starting or disconnecting an IV line, physically caring for a patient on a CP regimen, disposing of body fluids from a patient on $\mathrm{CP}$, touching a container, disposing of drug waste, touching a drugcontaminated surface, and consuming food in an area where antineoplastic drugs are handled.

The self-administered questionnaire asked questions regarding a participant's gender, job title, previous training related to the safe handling of antineoplastic drugs and the usual degree of contact with antineoplastic drugs. The latter included whether the worker had a duty to handle antineoplastic drugs, whether the individual was assigned to work in an area where antineoplastic drugs are handled/prepared and/or administered and the percentage of time spent on a typical work shift handling, preparing and/or administering antineoplastic drugs. In addition, participants were asked a series of questions regarding their knowledge of safe handling procedures within their hospital as well as the risk of occupational exposure to antineoplastic drugs. These seven questions, equally weighted, resulted in a cumulative "knowledge" score (scores ranged from 0 to 7). The knowledge questions are in the study questionnaire (Section C) available from http://antineoexposure.spph.ubc.ca/healthcare-workers-exposure-antineoplasticdrugs/study-documents.

In addition, we gathered information regarding hospital characteristics: the hospital type (acute care or cancer treatment); whether the hospital had an isolated drug preparation area (yes or no); and where antineoplastic drugs were initially delivered to the facility (shipping/receiving department or direct to the pharmacy).

$\underline{\text { Statistical Analysis }}$ 
Both untransformed and log-transformed (base e) data were used to examine the distribution of the urinary $\mathrm{CP}$ concentration levels. When log-transformed, the measurements exhibited more of a normal distribution than the corresponding untransformed data. Summary statistics (arithmetic mean (AM), geometric mean (GM), geometric standard deviation (GSD), $75^{\text {th }}$ percentile, maximum, and proportion greater than LOD) were used to describe the data. Summaries of the urinary CP concentration levels were stratified by various independent variables from the survey instruments. "No" and "don't know" responses to pertinent questions from either of the two survey tools were combined into one category and compared with "yes" responses to the same question.

The urinary concentrations measured in the same subjects in the repeated sampling rounds were summarized separately and compared using a paired t-test and Pearson correlation coefficient.

To determine which factors were related to the urinary CP concentrations (ln-transformed), while controlling for the repeated measures, linear mixed effect models were performed with subject and hospital as random effects. Initial screening models included only one fixed effect each. The fixed effects included individual characteristics (job title, gender, duty to handle antineoplastic drugs, percentage of time handling antineoplastic drugs, working in an area where antineoplastic drugs are handled, and received safe drug handling training); whether $\mathrm{CP}$ was contacted during the work shift; the dermal concentration levels from a previous study of ours (Hon et al. 2014b); the number of types of contact with CP during the work shift; the participants' knowledge score; and hospital characteristics (hospital type, department, if facility had an isolated preparation room and where antineoplastic drugs are initially delivered). All independent variables (fixed effects) with $\mathrm{p}<0.25$ from the screening analyses were then offered in one linear mixed effects regression and those variables with $\mathrm{p}<0.05$ were retained in the final model. A random effects 
only (hospital, subject) model was also run to examine the variance components. All analyses were performed using TIBCO Spotfire S+ 8.2 for Windows (TIBCO Software Inc.).

\section{Data less than the limit of detection}

As with the dermal and surface concentration levels reported in our previous studies (Hon et al. 2013; Hon et al. 2014b), a large proportion of urine samples had concentrations less than the laboratory-determined limit of detection (Table 2). To address this, we explored ways to prevent the bias that will occur if samples less than the detection limit are omitted or if inappropriate quantitative values are assigned to them (Helsel 2009). Laboratory-calculated concentrations below the method limit of detection were available to us. Although these data have a lower signal-to-noise ratio than data above the detection limit, they were based on actual measurements rather than substitute values, a technique often used for exposure data below detection limits (Hornung and Reed 1990). As there are biases associated with using a substitute value (Ganser and Hewett 2010), we used the actual analytical data for concentrations below the detection limit in all data analyses.

\section{Results}

$\underline{\text { Study population and urinary } \mathrm{CP} \text { concentration levels }}$

In total, 103 healthcare workers agreed to participate in the study. It was not possible to calculate a true response rate because of the constraints of the recruitment methods dictated by hospital ethics boards; however, the proportions who participated of those contacted in the six facilities ranged from $55 \%$ to $76 \%$.

Of the 103 participants, 98 provided a second sample, for a total of 201 urine samples. Slightly more than half of the samples $(55 \%$ or $n=111)$ had CP levels greater than the LOD with a maximum reported concentration of $2.37 \mathrm{ng} / \mathrm{mL}$ (Table 1). The mean urinary $\mathrm{CP}$ concentration 
was $0.156 \mathrm{ng} / \mathrm{mL}$, the geometric mean $0.067 \mathrm{ng} / \mathrm{mL}$, the geometric standard deviation 3.18, and the $75^{\text {th }}$ percentile $0.129 \mathrm{ng} / \mathrm{mL}$. The $\mathrm{CP}$ concentrations in the two sampling rounds (Table 1 ) were positively correlated (Pearson $\mathrm{r}=0.145, \mathrm{p}<0.01$ ), but also differed significantly between the two sampling rounds $(\mathrm{p}<0.01$, paired $\mathrm{t}-\mathrm{test})$.

Characteristics of the study population and concentration by individual factors

A summary of jobs, genders and other characteristics of the study population can be found in Table 2. Also shown in the table are urinary CP concentrations stratified by these characteristics. The job category with the highest average urinary drug concentration was the unit clerks. The job category with the largest proportion of samples exceeding the LOD were those classified as "others" who work in the drug administration unit but are not responsible for administering the drugs to patients (i.e. volunteers, oncologists, ward aides and dieticians). Every job category examined had sample results above the LOD of $0.05 \mathrm{ng} / \mathrm{mL}$ with the maximum reported urinary concentration level originating from a pharmacy technician.

Participants who had a duty to handle antineoplastic drugs and those who handled these agents for more than $25 \%$ of their shift had higher GMs as well as a larger proportion of samples above the LOD. Workers who had received safe drug handling training in the past had lower average $\mathrm{CP}$ concentrations as well as a lower proportion of samples above the LOD than those who had never received training.

Participants who were not certain if they contacted CP during the shift had a higher proportion of samples above the LOD as well as had a higher average concentration level than those who indicated that they had contacted $\mathrm{CP}$ at least once on their shift (Table 2). However, there was no association between urinary $\mathrm{CP}$ levels and the number of types of contact with $\mathrm{CP}$ on the work shift (Table 3). 
Also shown in Table 3, there was no association between a worker's knowledge score and their resulting urinary concentration levels. Further, there was no correlation between the dermal wipe sample results in our previous study (Hon et al. 2014b) and the urinary CP concentrations reported in the current study.

\section{Concentration levels by hospital characteristics}

A review of Table 4 indicates that healthcare workers from the cancer centre had a higher proportion of samples above the LOD and a higher average CP concentration compared to their counterparts who work at an acute care setting.

Of the three departments examined, the "other" departments not involved in either drug preparation or drug administration had the highest arithmetic mean urinary concentration level. Facilities with an isolated drug preparation room had a higher proportion of samples exceeding the LOD and the maximum reported concentration level was from this type of facility. In facilities where the drugs are initially delivered to the shipping/receiving department, as opposed to the pharmacy directly, a higher proportion of samples were above the LOD and the average urinary concentration level was higher.

Linear mixed effects regression

Five independent variables had $\mathrm{p}<0.25$ in the screening analyses: 1) job title, 2) whether the participant had a duty to handle antineoplastic drugs, 3) whether the participant received training,

4) hospital type and 5) department. All other independent variables had p >0.25.

These five independent variables were offered as fixed effects in a linear mixed effects regression (with subject and hospital as random effects) to examine associations with urinary $\mathrm{CP}$ concentration. Two fixed effect variables remained in the final model: whether a participant had a duty to handle and whether a participant had ever received training (Table 5). Participants who 
had a duty to handle antineoplastic drugs had higher urinary CP concentrations. Participants who never had training regarding safe drug handling had higher CP levels in urine.

The final linear mixed effects regression model was as follows:

$$
y_{i j k}=\beta_{0}+\beta_{1} \text { Duty to handle } e_{i j k}+\beta_{2} \text { Training }_{\mathrm{ijk}}+\mathrm{u}_{\mathrm{i}}+\mathrm{v}_{\mathrm{ij}}+\mathrm{e}_{\mathrm{ijk}}
$$

where $\mathrm{y}$ is the ln-transformed $\mathrm{CP}$ concentration, $\mathrm{u}$ is a random effect for between-hospital variance, $v$ is a random effect for between-subject variance, e is within-subject random error, $i$ is hospital index, $\mathrm{j}$ is subject index, and $\mathrm{k}$ is measurement index.

In the random effects only model, almost all the variance was within subject (96.9\%), a negligible proportion between subject, and a small proportion between hospital (3.1\%).

\section{Discussion}

Our results demonstrate that Canadian healthcare workers involved in some capacity with the hospital medication system have detectable levels of antineoplastic drug, specifically nonmetabolized CP, in their urine. Slightly more than half the samples exceeded the LOD, but the proportion of samples above LOD ranged from $44 \%$ to $67 \%$ depending on the independent variable examined. All eight job categories examined had a maximum urinary concentration level above the LOD of $0.05 \mathrm{ng} / \mathrm{mL}$.

A review of the literature indicates that there is a great deal of variability with respect to antineoplastic drug concentration levels in the urine of healthcare workers. Some studies have reported little or no CP detected (Hedmer et al. 2008b; Mason et al. 2005; Turci et al. 2010; Sottani et al. 2011; Connor et al. 2010) while one reported that up to $80 \%$ of their samples exceeded the LOD (Burgaz et al. 1999). A research team in Italy concluded that there has been a reduction in the percentage of positive urine samples over time (Sottani et al. 2010). As we are 
the first to determine $\mathrm{CP}$ urine concentration amongst Canadian healthcare workers, future similar studies are suggested to determine any possible trends in exposure levels in our country. The difference in mean urinary concentration between our two sampling sessions was statistically significant. Our random effects only model demonstrated the high proportion of the variance in urine concentrations was within subjects. These findings are consistent with Sugiura et al.'s observation that urinary concentration levels vary considerably amongst healthcare workers within a study (Sugiura et al. 2011). Differences in urinary CP concentrations within and between studies are likely due to variations in $\mathrm{CP}$ use, handling and cleaning practices, personal protective equipment usage as well as variability in metabolic rates amongst individuals (Villarini et al. 2011; Hedmer et al. 2008b).

In the current study, participants from departments where drug preparation and drug administration do not take place (shipping/receiving, transport, nutrition and materials management) had the highest average urinary concentration levels. Similarly, workers who do not work in an area where antineoplastic drugs are regularly handled had the higher mean concentration. Not surprisingly then, when the results were stratified by job title, unit clerks had the highest average urinary $\mathrm{CP}$ concentration. This may be novel to some as pharmacists and nurses have long been considered the highest risk job categories for occupational exposure to antineoplastic drugs.(McDiarmid et al. 2010) However, the results of the current study support our previous findings that other workers within the hospital medication system, besides nurses and pharmacy personnel, may be at greater risk of exposure to antineoplastic drugs (Hon et al. 2013).

We found that participants who did not report having contact with CP during their work shift had a higher mean concentration level and a higher proportion of samples exceed the LOD compared 
to those who did report $\mathrm{CP}$ contact. This is of concern as long-term urinary excretion of $\mathrm{CP}$ (i.e. continuously positive urine samples) may signify continuous absorption of CP (Sugiura et al. 2011) This phenomenon may be attributed to the fact that workers could have been exposed through skin contact due to environmental contamination from drug handling activities the day before (Tanimura et al. 2009). Another possibility is the fact that the skin may serve as a reservoir whereby internal exposure may continue despite the cessation of external exposure (Bos et al. 1998). Given this, it is not surprising that we did not find a correlation between the urinary concentration levels and the number of $\mathrm{CP}$ contact types that a worker may have had during the shift. This finding is consistent with others who have reported that antineoplastic drug handling activities are not associated with urinary concentration levels (Favier et al. 2003; Pethran et al. 2003; Sessink et al. 1994; Connor et al. 2010).

Participants from facilities in which the drugs were delivered to the shipping/receiving department had a higher mean concentration level as well as a larger proportion of samples above LOD than participants at facilities where drugs by-pass shipping/receiving and are delivered directly to the pharmacy. We observed the same finding for drug concentration levels found on various work surfaces (Hon et al. 2013). It should be noted that the two matrices were collected at different times; albeit at the same participating facilities. To determine if an association exists, it is recommended to collect environmental samples concurrently with urine samples as the former can demonstrate how, where and possibly when contamination occurred while the biological sample indicates if exposure occurred (Turci et al. 2010).

There was no correlation between the dermal concentration levels that we reported previously (Hon et al. 2014b) and urinary concentration levels in the current study. This could be because we collected the hand wipes at a single point in time which is not reflective of a worker's 
exposure throughout the shift. It could also suggest that other routes of exposure (e.g., inhalation and accidental ingestion) as well as metabolism may play a role in the body burden of antineoplastic drugs.

We identified two variables that were statistically significant in the linear mixed effects regression model: (1) whether a worker had a duty to handle antineoplastic drugs, and (2) whether the worker had ever received safe drug handling training. Not surprisingly, individuals tasked with handling antineoplastic drugs had higher $\mathrm{CP}$ concentrations in their urine. The fact that workers who did not receive training had higher levels of urinary $\mathrm{CP}$ concentration lends support that training is an important administrative control to reduce the level of occupational exposure to antineoplastic drugs. However, we also found that one's level of knowledge related to the risks of antineoplastic drugs was not associated with urinary concentration levels. Further evaluation of training and its effectiveness in reducing the risk of exposure is therefore suggested.

With regards to the health risks associated with the urinary concentration levels reported in this study, a no-significant-risk level (NSRL) of $1000 \mathrm{ng} /$ day has been suggested for $\mathrm{CP}^{2}$ (Sargent et al. 2002). The NSRL is the level at which no increase in cancer risk and no adverse effects on organ systems or the developing fetus are expected (Sargent et al. 2002). Converting our results to ng/day based on the estimated 24-hr urinary output volume per participant, we found 10 observations (4.5\%) had exposure levels which exceeded the NSRL threshold. Of these ten samples greater than $1000 \mathrm{ng} /$ day, four were from nurses, two were from pharmacy technicians, one was from a pharmacist, two were from unit clerks and one was from an individual who worked in a drug administration unit but was not responsible for drug administration. The latter

\footnotetext{
${ }^{2}$ Assumed that "day" is equivalent to 24 hours
} 
two job categories reaffirms the notion that other healthcare workers, in addition to nurses and pharmacy personnel, are at risk of occupational exposure to antineoplastic drugs.

Limitations of this study are described here. We collected a hand wipe sample from every participant immediately prior to collecting urine samples. As the skin is a possible reservoir source for the uptake of $\mathrm{CP}$, our results may underestimate the $\mathrm{CP}$ concentrations in urine. However, hand wipe sampling is akin to handwashing and virtually all of our participants washed their hands at least once prior to sample collection, so the extent of underestimation of urinary concentrations from the additional wiping via our study is not expected to be large. Housekeepers (cleaners) were not participants in the study because the contract company that employed the housekeepers declined to participate. This cohort is known to be at risk of exposure because they are responsible for removing the antineoplastic drug waste containers from the various departments at each facility as well as cleaning of patient lavatories (Fransman, et al. 2005; Hedmer et al. 2008b). We measured for only one antineoplastic drug, but healthcare workers are potentially exposed to many others. Lastly, several of the independent variables were gathered via self-reports and we cannot confirm their accuracy.

\section{Conclusions}

This study confirmed the presence of antineoplastic drug, specifically non-metabolized CP, in the urine of a broad range of healthcare workers who work at Canadian hospitals. This is consistent with the literature with respect to the fact that, despite the implementation of protective guidelines, occupational uptake of antineoplastic drugs is still occurring (Suspiro and Prista 2011). These findings also reaffirm our notion that more healthcare workers than initially believed are potentially exposed to antineoplastic drugs (Hon et al. 2011; Hon et al., 2014b). We identified two factors associated with urinary concentration - if a worker has a duty to 
handle antineoplastic drugs and whether a worker received training on safe drug handling. This finding provides a basis for targeting interventions to reduce occupational exposure. To our knowledge, this is the first study based in Canada to identify determinants of urinary CP levels for a broad range of healthcare workers. We therefore suggest that similar occupational exposure studies in the future include all potentially exposed job categories that are involved in the hospital medication system. Furthermore, interventions to minimize the risk of occupational exposure to antineoplastic drugs should be broadly applied and not focussed strictly on pharmacy personnel and nurses.

\section{Acknowledgements}

The authors would like to thank the WorkSafeBC Research Secretariat for funding this project (RS2008-OG01). We also appreciate the time and cooperation of all participants and their respective facilities. We would like to recognize the assistance of the Dr. Winnie Chu from University of British Columbia who led the team responsible for sample analyses. We would like to acknowledge Jennifer Shum, Pearl Siganporia and Sarah Chiarello who helped with sample collection efforts.

\section{Conflict of Interest}

The authors declare that they have no conflict of interest. 


\section{References}

Bos PM, Brouwer DH, Stevenson H, Boogaard PJ, de Kort WL, van Hemmen JJ (1998). Proposal for the Assessment of Quantitative Dermal Exposure Limits in Occupational Environments: Part 1. Development of a Concept to Derive a Quantitative Dermal Occupational Exposure Limit. Occ Environ Med 55 (12): 795-804.

Burgaz S, Karahalil B, Bayrak P, et al. (1999). Urinary Cyclophosphamide Excretion and Micronuclei Frequencies in Peripheral Lymphocytes and in Exfoliated Buccal Epithelial Cells of Nurses Handling Antineoplastics. Mutation Res 439 (1): 97-104.

Connor TH, DeBord DG, Pretty JR, et al. (2010). Evaluation of antineoplastic drug exposure of health care workers at three university-based US cancer centers. J Occup Environ Med 52(10): 1019-1027.

Connor TH and McDiarmid MA (2006). Preventing Occupational Exposures to Antineoplastic Drugs in Health Care Settings. CA:A Cancer Journal for Clinicians 56(6): 354-65.

De Jonge ME, Huitema ADR, Rodenhuis S, Beijnen JH (2005). Clinical Pharmacokinetics of Cyclophosphamide. Clinical Pharmacokinetics 44(11): 1135-64.

Dranitsaris G, Johnston M, Poirier S, et al. (2005). Are Health Care Providers Who Work with Cancer Drugs at an Increased Risk for Toxic Events? A Systematic Review and Meta-Analysis of the Literature. J Oncol Pharm Pract 11(2): 69-78.

Favier B, Gilles L, Desage M, Latour JF (2003). Analysis of Cyclophosphamide in the Urine of Antineoplastic Drugs Handlers. Bulletin Du Cancer 90(10): 905-9.

Fransman W, Vermeulen R, Kromhout H (2005). Dermal Exposure to Cyclophosphamide in Hospitals during Preparation, Nursing and Cleaning Activities. Int Arch Occup Environ Health 78(5): 403-12.

Ganser GH and Hewett P (2010). An accurate substitution method for analyzing censored data. J Occup Environ Hyg 7(4): 233-244.

Hedmer M, Höglund P, Cavallin-Ståhl E, Albin M, Jönsson BAG (2008a). Validation of Urinary Excretion of Cyclophosphamide as a Biomarker of Exposure by Studying Its Renal Clearance at High and Low Plasma Concentrations in Cancer Patients. Int Arch Occup Environ Health 81(3): 285-93.

Hedmer M, Tinnerberg H, Axmon A, Joensson BAG (2008b). Environmental and Biological Monitoring of Antineoplastic Drugs in Four Workplaces in a Swedish Hospital. Int Arch Occup Environ Health 81(7): 899-911.

Helsel D (2010). Much ado about next to nothing: incorporating nondetects in science. Ann Occup Hyg 54(3): 257-262. 
Hon C-Y, Astrakianakis G, Danyluk Q, Chu WC (2011a). Pilot Evaluation of Dermal Contamination by Antineoplastic Drugs among Hospital Pharmacy Personnel. Can J Hosp Pharm 64(5): 327-32.

Hon C-Y, Barzan C, Astrakianakis G. (2014a). Identification of Knowledge Gaps Regarding Healthcare Workers' Exposure to Antineoplastic Drugs: Review of Literature, North America versus Europe. Safety and Health at Work. In print doi:10.1016/j.shaw.2014.06.001.

Hon C-Y, Teschke K, Chu W, Demers P, Venners S (2013). Antineoplastic Drug Contamination of Surfaces Throughout the Hospital Medication System in Canadian Hospitals. J Occup Environ Hyg 10(7): 374-83.

Hon C-Y, Teschke K, Chua PPS, Venners S, Nakashima L (2011b). Occupational Exposure to Antineoplastic Drugs: Identification of Job Categories Potentially Exposed throughout the Hospital Medication System. Saf Health Work 2(3): 273.

Hon C-Y, Teschke K, Demers PA, Venners S (2014b). Antineoplastic Drug Contamination on the Hands of Employees Working throughout the Hospital Medication System. Ann Occup Hyg. In print doi: 10.1093/annhyg/meu019

Hornung R and Reed L (1990) Estimation of Average Concentration in the Presence of Nondetectable Values. Am Ind Hyg J 5(1): 46-51.

Jakubowski Marek and Trzcinka-Ochocka M (2005) Biological Monitoring of Exposure: Trends and Key Developments. J Occup Health 47(1): 22-48.

Mason HJ, Blair S, Sams C, Jones K, Garfitt SJ, Cuschieri MJ, Baxter PJ (2005). Exposure to antineoplastic drugs in two UK hospital pharmacy units. Ann Occup Hyg. 49(7): 603-610.

McDiarmid MA, Oliver MS, Roth TR, Rogers B, Escalante C (2010) Chromosome 5 and 7 Abnormalities in Oncology Personnel Handling Anticancer Drugs. J Occup Environ Med 52 (10):1028-34.

NIOSH (2004) Preventing Occupational Exposure to Antineoplastic and Other Hazardous Drugs in Health Care Settings (2004-165). Accessed 30 November 2011.

http://www.cdc.gov/niosh/docs/2004-165/.

Pethran A, Schierl R, Hauff K, et al., (2003) Uptake of Antineoplastic Agents in Pharmacy and Hospital Personnel. Part I: Monitoring of Urinary Concentrations. Int Arch Occup Environ Health 76(1): 5-10.

Ritchie MA, McAdams C, Fritz N (2000) Exposure Risk in the Handling and Administration of Chemotherapy Agents: A Review and Synthesis of the Literature. Online J Knowledge Synthesis Nurs 7(1): 51-60. 
Sargent EV, Naumann BD, Dolan DG, Faria EC, Schulman L (2002). The Importance of Human Data in the Establishment of Occupational Exposure Limits. Human Ecol Risk Assess 8: 805-22.

Sessink PJ, Van de Kerkhof MC, Anzion RB, Noordhoek J, Bos RP (1994). Environmental Contamination and Assessment of Exposure to Antineoplastic Agents by Determination of Cyclophosphamide in Urine of Exposed Pharmacy Technicians: Is Skin Absorption an Important Exposure Route? Arch Environ Health 49(3): 165-69.

Sottani C, Porro B, Comelli M, Imbriani M, Minoia C (2010). An analysis to study trends in occupational exposure to antineoplastic drugs among health care workers. J Chromat B 878(27), 2593-2605.

Sottani C, Porro B, Imbriani M, Minoia C (2011). Occupational Exposure to Antineoplastic Drugs in Four Italian Health Care Settings. Tox Letters 213(1):107-115.

Sugiura S, Asano M, Kinoshita K, Tanimura M, Nabeshima T (2011a). Risks to Health Professionals from Hazardous Drugs in Japan: A Pilot Study of Environmental and Biological Monitoring of Occupational Exposure to Cyclophosphamide. J Oncol Pharm Pract 17(1): 14-19.

Sugiura S, Nakanishi H, Asano M, et al. (2011b). Multicenter Study for Environmental and Biological Monitoring of Occupational Exposure to Cyclophosphamide in Japan. J Oncol Pharm Pract 17(1): 20-28.

Suspiro A, and Prista J (2011). Biomarkers of Occupational Exposure Do Anticancer Agents: A Minireview. Tox Letters 207(1): 42-52.

Tanimura M, Yamada K, Sugiura S, et al. (2009). An Environmental and Biological Study of Occupational Exposure to Cyclophosphamide in the Pharmacy of a Japanese Community Hospital Designated for the Treatment of Cancer. J Health Sci 55: 750-56.

Turci R, Minoia C, Sottani C, et al. (2010). Occupational Exposure to Antineoplastic Drugs in Seven Italian Hospitals: The Effect of Quality Assurance and Adherence to Guidelines. J Oncol Pharm Pract 17(4): 320-332.

US EPA (2011). Series 875 - Chemical Safety and Pollution Prevention. Accessed 26 October 2011. http://www.epa.gov/ocspp/pubs/frs/publications/Test_Guidelines/series875.htm.

Villarini M, Dominici L, Piccinini R, et al. (2011). Assessment of Primary, Oxidative and Excision Repaired DNA Damage in Hospital Personnel Handling Antineoplastic Drugs. Mutagenesis 26(3): 359-69. 
Table 1: Urinary concentrations of CP (in $\mathrm{ng} / \mathrm{mL}$ ) overall and in each of the two sampling rounds

\begin{tabular}{lccccccc}
\multicolumn{1}{c}{ Subcategory } & Sample Size & \% $>$ LOD & AM & GM & GSD & 75th \% & Max \\
\hline Overall & 201 & 55 & 0.156 & 0.067 & 3.18 & 0.129 \\
Sampling round 1 & 103 & 40 & 0.082 & $<$ LOD & 2.59 & 0.086 & 0.74 \\
Sampling round 2 & 98 & 71 & 0.234 & 0.095 & 3.49 & 0.190 & 2.37 \\
\hline
\end{tabular}


Table 2: Urinary concentrations of $\mathrm{CP}$ (in $\mathrm{ng} / \mathrm{mL}$ ) stratified by individual characteristics and CP contact during shift: summary statistics and $\mathrm{p}$-values for each fixed effect variable in screening ${ }^{\mathrm{a}}$ analyses

\begin{tabular}{|c|c|c|c|c|c|c|c|c|c|c|}
\hline Fixed effect variable & Categories & \multicolumn{2}{|c|}{ Sample size } & $\begin{array}{l}\%> \\
\text { LOD }\end{array}$ & $\mathbf{A M}$ & GM & GSD & 75th \% & Max & p-value \\
\hline \multirow{8}{*}{ Title } & Pharmacist & 16 & 16 & 44 & 0.089 & $<\mathrm{LOD}$ & 2.79 & 0.095 & 0.67 & Reference \\
\hline & Pharmacy receiver & 6 & 6 & 58 & 0.115 & 0.064 & 2.79 & 0.096 & 0.61 & 0.46 \\
\hline & Pharmacy technician & 21 & 21 & 57 & 0.170 & 0.065 & 3.23 & 0.125 & 2.37 & 0.28 \\
\hline & Porter & 6 & 4 & 50 & 0.085 & 0.063 & 2.25 & 0.123 & 0.25 & 0.35 \\
\hline & Nurse & 29 & 28 & 56 & 0.185 & 0.075 & 3.45 & 0.167 & 1.83 & 0.08 \\
\hline & Transport & 4 & 4 & 63 & 0.134 & 0.057 & 3.54 & 0.079 & 0.72 & 0.74 \\
\hline & Unit Clerk & 11 & 10 & 55 & 0.197 & 0.091 & 3.54 & 0.261 & 1.07 & 0.09 \\
\hline & $\begin{array}{l}\text { Other (volunteer, oncologist, } \\
\text { ward aide, dietician) }\end{array}$ & 10 & 9 & 67 & 0.182 & 0.065 & 3.36 & 0.095 & 2.01 & 0.59 \\
\hline \multirow{2}{*}{ Gender } & Female & 82 & 79 & 55 & 0.158 & 0.067 & 3.25 & 0.141 & 2.01 & Reference \\
\hline & Male & 21 & 19 & 55 & 0.151 & 0.064 & 2.96 & 0.110 & 2.37 & 0.89 \\
\hline \multirow{2}{*}{ Duty to handle? } & Yes & 82 & 80 & 56 & 0.160 & 0.069 & 3.16 & 0.130 & 2.37 & Reference \\
\hline & No & 21 & 18 & 51 & 0.142 & 0.056 & 3.29 & 0.117 & 2.01 & 0.18 \\
\hline \multirow{2}{*}{$\begin{array}{l}\text { Percentage of time } \\
\text { handling }\end{array}$} & $<25 \%$ & & 66 & 54 & 0.170 & 0.066 & 3.39 & 0.113 & 1.83 & Reference \\
\hline & $>25 \%$ & & 32 & 57 & 0.152 & 0.076 & 2.95 & 0.156 & 2.37 & 0.47 \\
\hline \multirow{2}{*}{$\begin{array}{l}\text { Work in an area where } \\
\text { antineoplastic drugs are } \\
\text { handled? }\end{array}$} & Yes & 72 & 72 & 56 & 0.150 & 0.067 & 3.09 & 0.128 & 2.01 & Reference \\
\hline & No & 31 & 26 & 54 & 0.173 & 0.066 & 3.45 & 0.130 & 2.37 & 0.93 \\
\hline \multirow{2}{*}{ Received training? } & Yes & 52 & 52 & 47 & 0.129 & 0.057 & 3.01 & 0.108 & 1.83 & Reference \\
\hline & No & 51 & 46 & 64 & 0.185 & 0.080 & 3.30 & 0.160 & 2.37 & 0.06 \\
\hline \multirow{2}{*}{$\begin{array}{l}\text { Contact with } \mathrm{CP} \text { on this } \\
\text { shift? }\end{array}$} & No/Don't know & 73 & 71 & 58 & 0.181 & 0.071 & 3.37 & 0.133 & 2.37 & Reference \\
\hline & Yes & 30 & 27 & 49 & 0.095 & 0.057 & 2.70 & 0.118 & 0.55 & 0.27 \\
\hline
\end{tabular}

${ }^{a}$ Linear mixed effects models of In-transformed urinary CP concentration, each model with a single fixed effect variable and hospital and subject as random effects 
Table 3: Linear mixed effect models of urinary CP concentration levels (ln-transformed) in screening ${ }^{\mathrm{a}}$ analyses of various continuous independent variables

\section{Fixed effect variable}

Intercept

Dermal concentration $^{\mathrm{b}}$

$-2.697$

Coefficient

SE

Number of types of CP contact on shift ${ }^{\mathrm{c}}$

$-2.660$

$-0.018$

$-2.565$

$-0.066$

0.032

0.062

p-value

Knowledge score ${ }^{\mathrm{d}}$

$-0.024$

0.043

0.57

${ }^{a}$ Each model with a single fixed effect variable and hospital and subject as random effect

${ }^{b}$ Mean $=0.36 \mathrm{ng} /$ wipe; range $<0.36$ to $22.8 \mathrm{ng} /$ wipe

Mean $=2$; range 0 to 7

${ }^{d}$ Mean $=5.8 ;$ range 0 to 7 (perfect $)$

Table 4: Urinary concentration levels of CP (in $\mathrm{ng} / \mathrm{mL}$ ) stratified by hospital characteristics: summary statistics and p-values for each fixed effect variable in screening ${ }^{\mathrm{a}}$ analyses

\begin{tabular}{|c|c|c|c|c|c|c|c|c|c|c|}
\hline Fixed effect variable & Categories & $\begin{array}{r}\text { Sam } \\
\text { Rnd 1 } \\
\end{array}$ & $\begin{array}{l}\text { Size } \\
\text { Rnd } 2 \\
\end{array}$ & $\begin{array}{l}\%> \\
\text { LOD }\end{array}$ & $\mathbf{A M}$ & GM & GSD & $\begin{array}{c}\text { 75th } \\
\%\end{array}$ & $\operatorname{Max}$ & p-value \\
\hline \multirow{2}{*}{ Type } & Acute Care & 82 & 77 & 52 & 0.137 & 0.060 & 3.03 & 0.113 & 2.01 & Reference \\
\hline & Cancer Centre & 21 & 21 & 67 & 0.228 & 0.097 & 3.58 & 0.212 & 2.37 & 0.12 \\
\hline \multirow{3}{*}{ Department } & Drug administration & 40 & 46 & 59 & 0.169 & 0.076 & 3.23 & 0.167 & 1.83 & Reference \\
\hline & Other $^{\mathrm{b}}$ & & 9 & 55 & 0.184 & 0.064 & 3.50 & 0.112 & 2.01 & 0.68 \\
\hline & Pharmacy & & 43 & 51 & 0.134 & 0.058 & 3.04 & 0.106 & 2.37 & 0.16 \\
\hline \multirow{2}{*}{$\begin{array}{l}\text { Isolated preparation } \\
\text { room }\end{array}$} & No & 22 & 19 & 49 & 0.157 & 0.062 & 3.37 & 0.107 & 1.83 & Reference \\
\hline & Yes & 81 & 79 & 57 & 0.156 & 0.068 & 3.15 & 0.129 & 2.37 & 0.78 \\
\hline $\begin{array}{l}\text { Where drugs are initially } \\
\text { delivered }\end{array}$ & $\begin{array}{l}\text { Pharmacy } \\
\text { Shipping/receiving }\end{array}$ & $\begin{array}{l}45 \\
58\end{array}$ & $\begin{array}{l}42 \\
46\end{array}$ & $\begin{array}{l}52 \\
58\end{array}$ & $\begin{array}{l}0.133 \\
0.174\end{array}$ & $\begin{array}{l}0.061 \\
0.071\end{array}$ & $\begin{array}{l}3.15 \\
3.21\end{array}$ & $\begin{array}{l}0.131 \\
0.125\end{array}$ & $\begin{array}{l}1.83 \\
2.37\end{array}$ & $\begin{array}{c}\text { Reference } \\
0.53\end{array}$ \\
\hline
\end{tabular}

${ }^{a}$ Linear mixed effects models of ln-transformed urinary CP concentration, each model with a single fixed effect variable and hospital and subject as random effects

${ }^{b}$ Includes shipping/receiving, transport, nutrition and materials management 
Table 5: Coefficients, standard errors and p-values for final linear mixed effect regression model ${ }^{\text {a }}$ showing the factors related to urinary $\mathrm{CP}$ concentration (ln-transformed)

\begin{tabular}{lccc} 
Fixed effects variables & Coefficients & Standard Error & p-value \\
\hline Intercept & -2.813 & 0.143 & $<0.0001$ \\
Duty to handle - Yes & Reference & 0.218 & 0.045 \\
Duty to handle - No & -0.442 & \\
Received training - Yes & Reference & 0.172 & 0.018 \\
Received training - No & 0.414 & & \\
\hline${ }^{a}$ Linear mixed effects model of ln-transformed urinary CP concentration, with all fixed effect variables together and hospital and subject as random effects
\end{tabular}

${ }^{a}$ Linear mixed effects model of In-transformed urinary CP concentration, with all fixed effect variables together and hospital and subject as random effects 